\title{
Protein Kinase C Activity and Delayed Recovery of Sleep-Wake Cycle in Mouse Model of Bipolar Disorder
}

\author{
Eunsoo Moon ${ }^{1,2}$, Byeong-Moo Choe ${ }^{3}$, Je-Min Park ${ }^{1,2} \bowtie$, Young In Chung ${ }^{2}$, \\ Byung Dae Lee ${ }^{1,2}$, Jae-Hong Park ${ }^{3}$, Young Min Lee ${ }^{1,2}$, Hee Jeong Jeong ${ }^{1}$, \\ YongJun Cheon ${ }^{4}$, Yoonmi Choi', and Jeonghyun Park ${ }^{1}$ \\ 'Department of Psychiatry and Medical Research Institute, Pusan National University Hospital, Busan, Republic of Korea \\ ${ }^{2}$ Department of Psychiatry, Pusan National University School of Medicine, Yangsan, Republic of Korea \\ ${ }^{3}$ Department of Psychiatry, Dong-A University School of Medicine, Busan, Republic of Korea \\ ${ }^{4}$ Department of Psychiatry, Dongrae Hospital, Busan, Republic of Korea
}

Objective Previous studies reported the delayed recovery group after circadian rhythm disruption in mice showed higher quinpiroleinduced locomotor activity. This study aimed to compare not only Protein Kinase C (PKC) activities in frontal, striatal, hippocampus and cerebellum, but also relative PKC activity ratios among brain regions according to recovery of circadian rhythm.

Methods The circadian rhythm disruption protocol was applied to eight-week-old twenty male Institute Cancer Research mice. The circadian rhythm recovery patterns were collected through motor activities measured by Mlog system. Depressive and manic proneness were examined by forced swim test and quinpirole-induced open field test respectively. Enzyme-linked immunosorbent assay was employed to measure PKC activities.

Results The delayed recovery group presented greater locomotor activities than the early recovery group ( $\mathrm{p}=0.033)$. The delayed recovery group had significantly lower frontal PKC activity than the other $(\mathrm{p}=0.041)$. The former showed lower frontal/cerebellar PKC activity ratio $(\mathrm{p}=0.047)$ but higher striatal/frontal $(\mathrm{p}=0.038)$ and hippocampal/frontal $(\mathrm{p}=0.007) \mathrm{PKC}$ activities ratios than the latter.

Conclusion These findings support potential mechanism of delayed recovery after circadian disruption in bipolar animal model could be an alteration of relative PKC activities among mood regulation related brain regions. It is required to investigate the PKC downstream signaling related to the delayed recovery pattern.

Psychiatry Investig 2018;15(9):907-913

Key Words Bipolar disorder, Circadian rhythm, Quinpirole, Protein Kinase C, Open field test.

\section{INTRODUCTION}

Abnormalities of the circadian rhythm might be related to the pathogenesis of bipolar disorder. Circadian rhythms of physiological, neuroendocrinological and neurochemical factors were disrupted in bipolar patients. ${ }^{1}$ Circadian rhythm

\footnotetext{
Received: April 3, 2018 Revised: May 9, 2018

Accepted: May 23, 2018

$\triangle$ Correspondence: Je-Min Park, MD, PhD

Department of Psychiatry and Medical Research Institute, Pusan National University Hospital, Pusan National University School of Medicine, 179 Gudeokro, Seo-gu, Busan 49241, Republic of Korea

Tel: +82-51-240-7306, Fax: +82-51-248-3648, E-mail: jmback@pusan.ac.kr

The results of this study were presented at the 19th Annual Conference of The International Society for Bipolar Disorders, May 4-7, 2017, Washington DC, USA.

(a) This is an Open Access article distributed under the terms of the Creative Commons Attribution Non-Commercial License (https://creativecommons.org/licenses/bync/4.0) which permits unrestricted non-commercial use, distribution, and reproduction in any medium, provided the original work is properly cited.
}

disruption has a great influence on the course of bipolar disorder. ${ }^{2}$ Furthermore, remitted bipolar patients showed abnormalities in sleep patterns such as increased sleep duration, elongated sleep latency, frequent wake after sleep onset, great percentage of rapid eye movement sleep, decreased sleep efficiency and more night-to-night variability of sleep patterns. ${ }^{3}$ Several genetic studies also showed that polymorphisms of circadian clock genes like CLOCK, PER3, ARNTL (BmaL1), and TIMELESS were significantly associated with bipolar disorder. ${ }^{4,5}$

Abnormality of Protein Kinase C (PKC) signaling is found in both bipolar disorder and circadian rhythm. Several studies showed changes of PKC activity in platelet of peripheral blood and postmortem brain tissues in patients with bipolar disorder. ${ }^{6-10}$ Furthermore, these changes of PKC activity in bipolar patients were normalized by long-term treatment of lithium or valproate. ${ }^{6,7}$ PKC signaling pathway is also in- 
volved in the regulation of circadian rhythm. PKC $\alpha$ has a great influence on resetting circadian rhythm through the phosphorylation of lysine-specific demethylase 1 that modulates the transcription of an Enhancer Box by Clock-BmaL heterocomplex, a key component in transcription-translation loop of circadian clock. ${ }^{11}$ PKC might be related to phase shifting and light entrainment of circadian rhythm through the modulation of post-translational mechanism. ${ }^{12}$

Although abnormalities of PKC signaling systems were studied in amphetamine-induced animal model and sleep deprivation model of bipolar disorder, these studies did not investigate the relatedness between PKC signaling abnormalities and circadian rhythm instability in bipolar animal model. ${ }^{13-15}$ There were two studies about circadian rhythm instability as bipolar animal model. ${ }^{16,17}$ However, PKC abnormalities were not examined in these studies. It could be meaningful to examine the changes of PKC activity in this circadian rhythm instability model. It is also needed to investigate PKC activities in various brain regions related to mood regulation and examine the interactions of PKC activities among these brain regions. ${ }^{18-20}$ This study aimed to compare not only PKC activities in frontal, striatal, hippocampus and cerebellum, but also relative $\mathrm{PKC}$ activity ratios among brain regions, between two groups that are classified into according to recovery of circadian rhythm.

\section{METHODS}

\section{Animals}

Twenty male ICR mice (Institute of Cancer Research, age of 8 weeks, weight 33-36 gm) were used in this study. All mice were housed individually in cages under a 12 -hour artificial light-dark cycle at a constant temperature $20 \pm 1^{\circ} \mathrm{C}$ and relative humidity of $40-60 \%$. Mice were entrained to the normal DL 12:12 cycle (Dark phase: lights off from 6 PM to 6 AM; Light phase: lights on from $6 \mathrm{AM}$ to $6 \mathrm{PM}$ ) at least for one week prior to the start of all experiments. Food and water were provided ad libitum.

\section{Experimental procedure}

After one week of entrainment to the DL 12:12 cycle, the circadian rhythm was disrupted by abruptly shifting of lighting condition to the LD 12:12 cycle (Light phase: lights on from 6 PM to 6 AM; Dark phase: light off from 6 AM to 6 PM). Recovery patterns of normal sleep-wake cycle after the disruption of circadian rhythm were classified into the early and delayed recovery groups. After two weeks of $\mathrm{LD}$ phase, all mice were reentrained to original DL 12:12 cycle. At the age of 14 weeks, after full recovery of sleep-wake cycle, forced swim test was performed in dark phase. At the age of 16 weeks, after two weeks from forced swim test, locomotor activities in the open field were measured. Mice were adapted in the open field for 30 minutes and locomotor activities in the open field were assessed for 60 minutes after the injection of quinpirole. Within 2 hours after the open field test, mice were sacrificed by rapid decapitation for measuring PKC activity. The characteristics of forced swim test, quinpirole-induced locomotor activities in the open field, PKC activities and relative PKC ratios of brain regions were compared between the early and delayed recovery groups. The experimental procedures and protocols used in this study were reviewed and approved by the Ethics Committee on Animal Research of Pusan National University (PNUH-2013-058).

\section{Measurements}

\section{Circadian rhythm disruption procedure}

As described previously, the circadian rhythm disruption protocol was composed of the entrainment phase and the disruption phase. After animals were entrained into the DL 12:12 cycle for at least one week, circadian rhythm was disrupted into the LD 12:12 cycle. The light was automatically controlled by Light Control System (Philips, Burlington, MA, USA) according to the circadian rhythm disruption protocol.

\section{Measurement of movement activity in each cage and recovery of circadian rhythm}

Movement activities of individual mice in each cage were measured in every second by Mlog system (Biobserve $\mathrm{GmbH}$, Bonn, Germany). Mlog system, which is custom-made infrared-based motion detectors, can measure gross body movements. The movement activity was converted to a voltage output, which was digitized. The magnitude of voltage output was directly related to the magnitude of the movements. Integrated values for body movement were stored as binary computer files. In the previous study, the agreement of locomotor activities measured by Mlog systems and classical video recording was adaptable (kappa index, 0.633). ${ }^{21}$ In this study, for assessment of the total movement activities in light and dark phase, signals for each 12 hour were summed. Assessment of recovery of circadian rhythm was performed by the comparison between summed movement activities in light and dark phase. Previous studies used the method determining the recovery time when total movement activities in dark phase are higher than those in light phase and thus the determined time does not strictly represented the fully recovery time..$^{16,17}$ Therefore, the author used new criteria for determining the full recovery time. The recovery time was defined by the time that the ratio of total movement activities in dark phase divided by total activities in light phase reached mean ratio of 


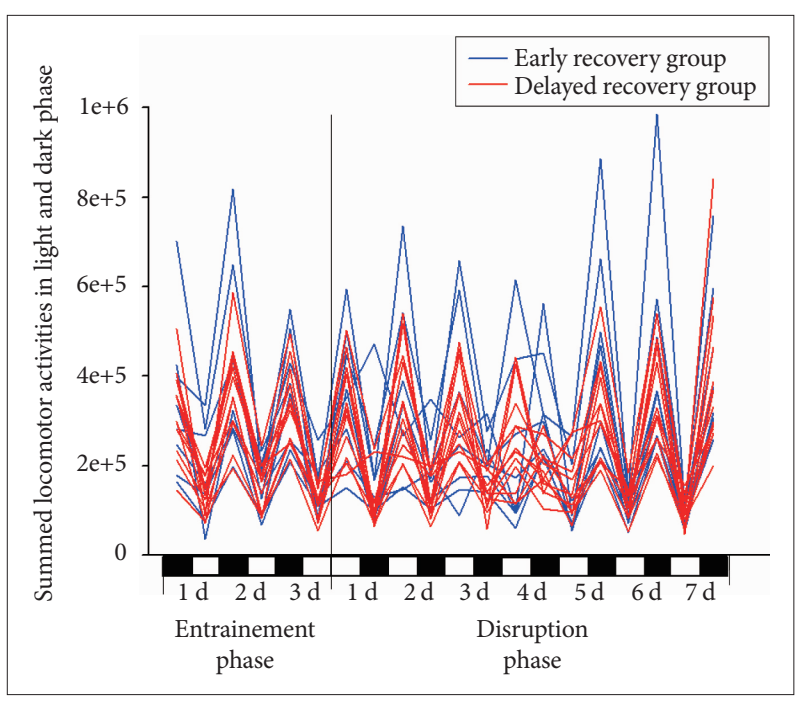

Figure 1. Recovery patterns according to time after the disruption of sleep-wake cycle in mouse. The blue line indicates summed locomotor activities in the early recovery group and the red line shows summed locomotor activities in the delayed recovery group.

baseline activities between dark and light phase. Mice were classified into two groups according to the recovery time of circadian rhythm, early recovery group and delayed recovery group, respectively. The recovery time of circadian rhythm ranged from 3 days to 7 days (5.6 \pm 1.1 ) (Figure 1). The median recovery time of circadian rhythms was 6 days. Nine mice were recovered in 6th day that is the mode of variable as recovery time of circadian rhythm. Mice recovered within 5 days were composed of 40 percentile among total subjects and mice recovered within 6 days consisted of 85 percentile. Therefore, in order to evenly divide the subjects, eight mice were classified into the early recovery group and twelve mice were classified into the delayed recovery group.

\section{Measurement of manic-like behaviors}

Manic-like behaviors were measured as quinpirole-induced locomotor activity in open field test. Locomotor activities in an open field were examined for 30 minutes before and for 60 minutes after quinpirole injection. Quinpirole $(0.5 \mathrm{mg} / \mathrm{kg})$ was diluted in deionized water and injected intraperitoneally during the dark period at 7-10 PM. The open field consisted of a square arena $(50 \times 50 \times 50 \mathrm{~cm})$ made of transparent acrylic board. At the start of each test, mouse was placed at the peripheral corner of the arena and videotaped. Raw data of locomotor activities for analysis were collected using Kinovea video tracking software program (Version 0.8.24. http://www. kinovea.org). Raw data of locomotor activities include $\mathrm{x}$ and $\mathrm{y}$ coordinate in recorded motion picture with time data linked to coordinates. For the validation of this analyzing program, the results examined by Kinovea video tracking software program were compared with the classical method to count the number of lines crossed by its front legs as a measurement of locomotor activity. ${ }^{16,17}$ Further data processing of raw data to analyze locomotor activities was performed using MATLAB R2013b (MathWorks, Natick, MA, USA).

\section{Measurement of depression-like behaviors}

Depression-like behaviors were measured as immobility time and latency to immobilization by the forced swim test. Forced swim test was developed as an animal model of depression. ${ }^{22,23}$ Mice were placed into a cylinder (diameter $20 \mathrm{~cm}$, height $30 \mathrm{~cm}$ ) filled with water (water height $15 \mathrm{~cm}$, water temperature $24 \pm 1^{\circ} \mathrm{C}$ ) for 6 minutes. Immobility time during last 4 minutes is used as a parameter reflecting depressive symptoms. ${ }^{23}$ Additionally, latency to immobilization was examined as an index of depression. ${ }^{24}$

\section{Brain tissues sampling}

After the last process of the planned experiment protocol, the brain samples of mice were collected through rapid decapitation, then tissues in the frontal cortex, hippocampus, striatum and cerebellum were dissected respectively. The samples were instantly frozen using liquid nitrogen, and kept at $-80^{\circ} \mathrm{C}$ until using for PKC activity measurement.

\section{Measurement of protein Kinase $\mathrm{C}$ activity}

PKC activities were examined in four regions of the brain PKC extraction buffer was added to the samples for the lysis of the cells. The supernatant of the lysates was collected for Bradford assay to measure the protein concentration. Finally, PKC activities of the brain sample extractions were measured using enzyme-linked immunosorbent assay (ELISA) using a PKC kinase activity kit (Enzo Life Sciences, Farmingdale, NY, USA). The PKC activity assay was specific for all types of PKC isoforms, and both inter- and intra-assay coefficients of variability are known to be less than $10 \%$.

\section{Statistical analysis}

The comparisons of locomotor activities in an open field, immobility time and latency to immobility in forced swim test, PKC activities and relative PKC activity ratios between early and delayed recovery groups were tested by independent $t$-test. All data were expressed as mean \pm SD. All statistical analyses were performed using SPSS for Windows (The Statistical Package for Social Science for Windows, version 18.0; SPSS Inc., Chicago, IL, USA) and level of statistical significance was set at $\mathrm{p}<0.05$. 


\section{RESULTS}

\section{Locomotor activities in the open field before and after quinpirole injection}

Locomotor activities in the open field before and after quinpirole injection were shown in Table 1. Though the delayed recovery group traveled longer distance in the open field test than the early recovery group, there was no statistically significant difference in the baseline locomotor activity between two groups ( $\mathrm{t}=-0.1934, \mathrm{p}=0.069)$. In the open field test after quinpirole injection, the delayed recovery group showed much longer distance traveled than the early recovery group $(\mathrm{t}=-2.361, \mathrm{p}=0.033)$. There were no statistically significant differences in latency to reach the central zone and time spent in the central zone between two groups. The delayed group significantly had longer distance traveled in central zone than the early group $(\mathrm{t}=-2.259, \mathrm{p}=0.040)$.

\section{Immobility time and latency to immobility in forced swim test}

In forced swim test, immobility times of the early and delayed recovery groups were $133.1( \pm 77.7)$ and $119.9( \pm 78.6)$ second, respectively. Latencies to immobility in two groups were $81.3( \pm 74.3)$ and $126.4( \pm 61.7)$, respectively (Table 1$)$. There were no significant differences of immobility time and latency to immobility between two groups (immobility time: $\mathrm{t}=0.370, \mathrm{p}=0.716$; latency to immobility: $\mathrm{t}=-1.479, \mathrm{p}=0.156$ ) (Table 1).

\section{Protein Kinase $\mathrm{C}$ activities in the brain regions}

The delayed recovery group had significantly lower frontal PKC activity than early recovery group $(\mathrm{t}=2.411, \mathrm{p}=0.041)$. However, there were no statistically significant differences of PKC activities in the striatal, hippocampal, and cerebellum between two groups (Table 2). When comparing relative PKC activities ratio by adjusting cerebellar PKC activities, frontal/

Table 1. Comparison of behavioral data in the open field test and forced swim test between the early recovery and the delayed recovery groups after disruption of the sleep-wake cycle

\begin{tabular}{lccc}
\hline \multicolumn{1}{c}{ Behavioral characteristics } & $\begin{array}{c}\text { Early recovery group } \\
(\text { mean } \pm \text { SD) }\end{array}$ & $\begin{array}{c}\text { Delayed recovery group } \\
\text { (mean } \pm \text { SD) }\end{array}$ & p value \\
\hline $\begin{array}{l}\text { Total distance traveled for 30 minutes } \\
\text { before quinpirole injection in OFT }\end{array}$ & $9,024.0 \pm 2,082.2$ & $11,383.8 \pm 2,989.2$ & -1.934 \\
$\quad \begin{array}{l}\text { Total distance traveled for 60 mins after } \\
\quad \text { quinpirole injection in OFT }\end{array}$ & $3,657.5 \pm 1,369.8$ & $6,616.0 \pm 4,003.7$ & -2.361 \\
Latency to reach the central zone & $92.6 \pm 97.2$ & $256.6 \pm 428.2$ & -1.278 \\
Time spent in central zone & $85.5 \pm 97.9$ & $117.5 \pm 102.5$ & -0.696 \\
Distance traveled in central zone & $240.3 \pm 214.7$ & $701.3 \pm 656.2$ & -2.259 \\
Immobility time in FST & $133.1 \pm 77.7$ & $119.9 \pm 78.6$ & 0.495 \\
Latency to immobility in FST & $81.3 \pm 74.3$ & $126.4 \pm 61.7$ & 0.370 \\
\hline
\end{tabular}

Statistics was tested by independent t-test. Distances were measured in centimeters and latency and time in seconds. OFT: open field test, FST: forced swim test

Table 2. Comparison of protein Kinase $\mathrm{C}$ activities and relative PKC ratios in the frontal, striatal, hippocampal, and cerebellar regions between the early and the delayed recovery groups

\begin{tabular}{|c|c|c|c|c|}
\hline $\mathrm{PKC}$ activities and relative $\mathrm{PKC}$ ratios & $\begin{array}{l}\text { Early recovery group } \\
(\text { mean } \pm \mathrm{SD})\end{array}$ & $\begin{array}{c}\text { Delayed recovery group } \\
(\text { mean } \pm \mathrm{SD})\end{array}$ & $\mathrm{t}$ & $\mathrm{p}$ value \\
\hline Frontal PKC activity & $1.54 \pm 0.64$ & $0.97 \pm 0.24$ & 2.411 & 0.041 \\
\hline Striatal PKC activity & $1.41 \pm 0.46$ & $1.46 \pm 0.43$ & -0.281 & 0.782 \\
\hline Hippocampal PKC activity & $1.21 \pm 0.33$ & $1.53 \pm 0.55$ & -1.612 & 0.124 \\
\hline Cerebellar PKC activity & $1.18 \pm 0.18$ & $1.19 \pm 0.32$ & -0.046 & 0.964 \\
\hline Frontal/cerebellar PKC activity ratio & $1.23 \pm 0.45$ & $0.85 \pm 0.27$ & 2.308 & 0.034 \\
\hline Striatal/cerebellar PKC activity ratio & $1.21 \pm 0.37$ & $1.26 \pm 0.31$ & -0.285 & 0.779 \\
\hline Hippocampal/cerebellar PKC activity ratio & $1.05 \pm 0.32$ & $1.33 \pm 0.54$ & -1.251 & 0.228 \\
\hline Striatal/frontal PKC activity ratio & $1.02 \pm 0.39$ & $1.57 \pm 0.61$ & -2.244 & 0.038 \\
\hline Hippocampal/frontal PKC activity ratio & $0.89 \pm 0.31$ & $1.69 \pm 0.80$ & -3.126 & 0.007 \\
\hline Striatal/hippocampal PKC activity ratio & $1.17 \pm 0.31$ & $1.01 \pm 0.27$ & 1.245 & 0.229 \\
\hline
\end{tabular}

Statistics was tested by independent t-test. PKC: Protein Kinase C 
cerebellar PKC activity ratio in the delayed recovery group showed significantly lower than that of the early recovery group ( $t=2.308, p=0.034)$. In contrast, there were no significant differences in the striatal/cerebellar and hippocampal/ cerebellar PKC activity ratios. Comparing relative PKC activities by adjusting frontal $\mathrm{PKC}$ activities, striatal/frontal and hippocampal/frontal PKC activity ratios in the delayed recovery group were significantly higher than those in the early recovery group (striatal/frontal $\mathrm{PKC}$ activity ratio: $\mathrm{t}=-2.244, \mathrm{p}=$ 0.038; hippocampal/frontal $\mathrm{PKC}$ activity ratio: $\mathrm{t}=-3.126, \mathrm{p}=$ 0.007). There was no significant difference in hippocampal/ striatal PKC activity ratio between two groups.

\section{DISCUSSION}

In this study, the delayed recovery group after circadian rhythm disruption showed higher quinpirole-induced locomotor activity than the early recovery group. This result is consistent with previous studies. ${ }^{17}$ Especially, the delayed group with circadian rhythm instability had longer distance traveled in the central zone than the early group. Activity in the central zone might be interpreted into explorative behavior and manic-like behavior. In contrast, there was no difference of immobility time during last 4 minutes in the forced swim test between two groups. Although the forced swim test was performed after the full recovery of circadian rhythm and before quinpirole injection in order to minimize confounding factors, the depressive proneness was not found in forced swim test. As similar to this result, the CLOCK gene mutant and sleep deprivation models for bipolar disorder showed only manic-like manifestations. The circadian rhythm instability model for bipolar disorder also might be fit to the manic proneness only. ${ }^{25,26}$ However, the D-binding protein knockout mice known as another circadian genetic model for bipolar disorder showed both depressive-like and manic-like behaviors in acute stress situation. ${ }^{27}$ Although the forced swim test is one of the most popular methods to screen the antidepressant efficacy, several researchers throw a doubt that the forced swim test is inappropriate as an animal model of depression. ${ }^{28}$ Other methods to measure depressive-like behaviors are required before concluding there are no relationship between delayed recovery of circadian rhythm and depressive proneness. ${ }^{29}$

In order to explore the common underlying neurobiological mechanisms related to delayed recovery of circadian rhythm and manic-like behaviors, the PKC activities and their relative ratios were examined in the frontal cortex, striatum, hippocampus and cerebellum. In this study, there was a significantly lower frontal PKC activity in the delayed group than the early recovery. However, several reports suggested that overactivation of platelet PKC is associated with manic state. ${ }^{6,9}$
In a postmortem study of bipolar patients, the frontal PKC activities were more increased than those of healthy controls. ${ }^{8}$ Only one study reported that PKC activities in platelets of pediatric bipolar patients were decreased and recovered after the treatment of mood stabilizers. ${ }^{30}$ It is intriguing that clinical studies showed early onset bipolar patients have abnormalities of circadian rhythm and sleep. ${ }^{31-34}$ The results of these studies indicated that the change of PKC activities might be different among subtypes of bipolar disorder.

This study showed meaningful interactions between the frontal and cerebellar regions. The frontal/cerebellar PKC activity ratios, however, were significantly decreased in the delayed group. The interactions between the frontal cortices and cerebellum have been studied for the autism. ${ }^{20}$ These interactions have a potential role of dopamine release in the frontal area. ${ }^{20,35}$ Dysfunctional connection between the frontal cortices and cerebellum can have a deleterious effect on the release of dopamine in the frontal area. Abnormalities of dopaminergic neurotransmission have been found in bipolar disorder, as well as schizophrenia. ${ }^{36}$ Numerous researches have been reported that bipolar patients have the abnormalities of frontal functions..$^{37,38}$ Decrease of the frontal/cerebellar PKC activity ratio may reflect the abnormality of dopaminergic neurotransmission caused by dysfunctional connection of cerebellofrontal circuit and the decrease of frontal function.

Meanwhile, there were significant differences in striatal/ frontal and hippocampal/frontal PKC activities ratio according to recovery patterns. The striatum is associated with the regulation of mood symptoms, as well as the coordination of motor and action planning, motivation, and decision making. ${ }^{39,40}$ As striatal PKC activities can be associated with modulation of dopaminergic neurotransmission, ${ }^{41}$ PKC might take important roles in mood regulation through modulation of dopaminergic neurotransmission. The hippocampus has important role on mood regulation, memory and spatial learning. ${ }^{13,42,43}$ There have been several lines of researches on the interaction between the frontal and limbic regions such as the striatum and hippocampus. ${ }^{44-46}$ This hypothesis of frontal-limbic dysregulation in bipolar disorder and major depression insists that the deactivation of the frontal area and activation of limbic system are associated with the pathogenesis of mood disorder. ${ }^{4-46}$ The findings that the relative activation of striatal and hippocampal PKC activities compared to frontal PKC activities are consistent with several studies on the hypothesis of fronto-limbic dysregulation and dopaminergic neurotransmission in bipolar disorder.

This study has several limitations. Firstly, only drug-induced locomotor activities and behavioral despair were measured to test bipolar-proneness in delayed recovery group. Other indices such as sucrose preference test and social activity are 
needed to explore on. Secondly, the author did not measure $\mathrm{PKC}$ isoforms and the downstream signaling of PKC necessary to explore the underlying biological mechanism. Thirdly, the residual stressful effect by forced swim test might have an influence on quinpirole-induced locomotor activities in open field test, though open field test was performed after 2 weeks of forced swim test for the minimization of the impact of forced swim test. However, previous study that performed open field test before forced swim test in opposite sequences showed the same results. Fourthly, this study only used forced swim test without depression-inducing stimuli. If future study use forced swim test in case of utilization of various types of stress as depression-inducing stimuli, forced swim test could provide the more meaningful findings in order to evaluate depressive proneness.

In conclusion, the results of support that delayed recovery after circadian rhythm disruption might be related to manic proneness. The results showed possible relationship between circadian instability and abnormalities of PKC signaling in a bipolar animal model. Especially, delayed recovery after circadian rhythm disruption may be related to a lower frontal PKC activity and imbalances of PKC activity in regions of frontal-cerebellum, striatal-cerebellum and hippocampalcerebellum. Further studies are needed to investigate depressive proneness in mice with delayed recovery patterns using other methodologies for examining depressive-like behavior. It is also required to examine the changes of PKC isoforms such as PKC $\alpha, \beta, \gamma$ and the downstream molecules such as MACKRS, GAP-43 in order to understand the specific molecular pathway of this circadian rhythm instability as a bipolar disorder animal model.

\section{REFERENCES}

1. Murray G, Harvey A. Circadian rhythms and sleep in bipolar disorder. Bipolar Disord 2010;12:459-472.

2. Malkoff-Schwartz S, Frank E, Anderson B, Sherrill JT, Siegel L, Patterson $\mathrm{D}$, et al. Stressful life events and social rhythm disruption in the onset of manic and depressive bipolar episodes: a preliminary investigation. Arch Gen Psychiatry 1998;55:702-707.

3. Geoffroy PA, Scott J, Boudebesse C, Lajnef M, Henry C, Leboyer M, et al. Sleep in patients with remitted bipolar disorders: a meta-analysis of actigraphy studies. Acta Psychiatr Scand 2015;131:89-99.

4. Serretti A, Benedetti F, Mandelli L, Lorenzi C, Pirovano A, Colombo C, et al. Genetic dissection of psychopathological symptoms: insomnia in mood disorders and CLOCK gene polymorphism. Am J Med Genet B Neuropsychiatr Genet 2003;121:35-38.

5. Shi J, Wittke-Thompson JK, Badner JA, Hattori E, Potash JB, Willour $\mathrm{VL}$, et al. Clock genes may influence bipolar disorder susceptibility and dysfunctional circadian rhythm. Am J Med Genet B Neuropsychiatr Genet 2008;147B:1047-1055.

6. Friedman E, Hoau Yan W, Levinson D, Connell TA, Singh H. Altered platelet protein kinase $\mathrm{C}$ activity in bipolar affective disorder, manic episode. Biol Psychiatry 1993;33:520-525.

7. Hahn CG, Umapathy, Wang HY, Koneru R, Levinson DF, Friedman E. Lithium and valproic acid treatments reduce $\mathrm{PKC}$ activation and re-
ceptor-G protein coupling in platelets of bipolar manic patients. J Psychiatr Res 2005;39:355-363.

8. Wang HY, Friedman E. Enhanced protein kinase C activity and translocation in bipolar affective disorder brains. Biol Psychiatry 1996;40: 568-575.

9. Wang HY, Markowitz P, Levinson D, Undie AS, Friedman E. Increased membrane-associated protein kinase $\mathrm{C}$ activity and translocation in blood platelets from bipolar affective disorder patients. J Psychiatr Res 1999;33:171-179.

10. Abrial E, Lucas G, Scarna H, Haddjeri N, Lambas-Senas L. A role for the PKC signaling system in the pathophysiology and treatment of mood disorders: involvement of a functional imbalance? Mol Neurobiol 2011; 44:407-419.

11. Nam HJ, Boo K, Kim D, Han DH, Choe HK, Kim CR, et al. Phosphorylation of LSD1 by PKCalpha is crucial for circadian rhythmicity and phase resetting. Mol Cell 2014;53:791-805.

12. Lee B, Almad A, Butcher GQ, Obrietan K. Protein kinase C modulates the phase-delaying effects of light in the mammalian circadian clock. Eur J Neurosci 2007;26:451-462.

13. Abrial E, Etievant A, Betry C, Scarna H, Lucas G, Haddjeri N, et al. Protein kinase $\mathrm{C}$ regulates mood-related behaviors and adult hippocampal cell proliferation in rats. Prog Neuropsychopharmacol Biol Psychiatry 2013;43:40-48.

14. Kantor L, Gnegy ME. Protein kinase C inhibitors block amphetaminemediated dopamine release in rat striatal slices. J Pharmacol Exp Ther 1998;284:592-598.

15. Szabo ST, Machado-Vieira R, Yuan P, Wang Y, Wei Y, Falke C, et al. Glutamate receptors as targets of protein kinase $\mathrm{C}$ in the pathophysiology and treatment of animal models of mania. Neuropharmacology 2009; 56:47-55.

16. Jung SH, Park JM, Moon E, Chung YI, Lee BD, Lee YM, et al. Delay in the recovery of normal sleep-wake cycle after disruption of the lightdark cycle in mice:a bipolar disorder-prone animal model? Psychiatry Investig 2014;11:487-491.

17. Kim SY, Moon E, Jeong HJ, Lee YM, Lee BD, Park JM. Delay in normalization of disrupted sleep-wake cycle in mice as a bipolar disorder-prone animal model (bipolar disorder-prone animal model). J Korean Neuropsychiatr Assoc 2016;55:209-214.

18. Huang FL, Yoshida Y, Nakabayashi H, Huang KP. Differential distribution of protein kinase $\mathrm{C}$ isozymes in the various regions of brain. J Biol Chem 1987;262:15714-15720.

19. Drevets WC. Neuroimaging studies of mood disorders. Biol Psychiatry 2000;48:813-829.

20. Ji L, Chauhan A, Chauhan V. Reduced activity of protein kinase C in the frontal cortex of subjects with regressive autism: relationship with developmental abnormalities. Int J Biol Sci 2012;8:1075-1084.

21. Jung MS, Lee YM, Moon E, Park JM. Prediction of Sleep-Awake State of Mouse by Mlog Locomotor Activity Analyzing System. Busan: Psychiatry, Pusan National University; 2015.

22. Porsolt RD, Le Pichon M, Jalfre M. Depression: a new animal model sensitive to antidepressant treatments. Nature 1977;266:730-732.

23. Can A, Dao DT, Arad M, Terrillion CE, Piantadosi SC, Gould TD. The mouse forced swim test. J Vis Exp 2012;(59):e3638.

24. Castagne V, Porsolt RD, Moser P. Use of latency to immobility improves detection of antidepressant-like activity in the behavioral despair test in the mouse. Eur J Pharmacol 2009;616:128-133.

25. Gessa GL, Pani L, Fadda P, Fratta W. Sleep deprivation in the rat: an animal model of mania. Eur Neuropsychopharmacol 1995;5 Suppl:89-93.

26. Roybal K, Theobold D, Graham A, DiNieri JA, Russo SJ, Krishnan V, et al. Mania-like behavior induced by disruption of CLOCK. Proc Natl Acad Sci U S A 2007;104:6406-6411.

27. Le-Niculescu H, McFarland MJ, Ogden CA, Balaraman Y, Patel S, Tan J, et al. Phenomic, convergent functional genomic, and biomarker studies in a stress-reactive genetic animal model of bipolar disorder and co-morbid alcoholism. Am J Med Genet B Neuropsychiatr Genet 2008; 
147B:134-166.

28. Molendijk ML, de Kloet ER. Immobility in the forced swim test is adaptive and does not reflect depression. Psychoneuroendocrinology 2015; 62:389-391.

29. Kato T, Kasahara T, Kubota-Sakashita M, Kato TM, Nakajima K. Animal models of recurrent or bipolar depression. Neuroscience 2016;321: 189-196.

30. Pandey GN, Ren X, Dwivedi Y, Pavuluri MN. Decreased protein kinase $\mathrm{C}$ (PKC) in platelets of pediatric bipolar patients: effect of treatment with mood stabilizing drugs. J Psychiatr Res 2008;42:106-116.

31. Perlis RH, Miyahara S, Marangell LB, Wisniewski SR, Ostacher M, DelBello MP, et al. Long-term implications of early onset in bipolar disorder: data from the first 1000 participants in the systematic treatment enhancement program for bipolar disorder (STEP-BD). Biol Psychiatry 2004;55:875-881.

32. Lofthouse N, Fristad M, Splaingard M, Kelleher K, Hayes J, Resko S. Web survey of sleep problems associated with early-onset bipolar spectrum disorders. J Pediatr Psychol 2008;33:349-357.

33. Dilsaver SC, Akiskal HS. "Mixed hypomania" in children and adolescents: is it a pediatric bipolar phenotype with extreme diurnal variation between depression and hypomania? J Affect Disord 2009;116:12-17.

34. Post RM, Leverich GS, Kupka RW, Keck PE Jr, McElroy SL, Altshuler LL, et al. Early-onset bipolar disorder and treatment delay are risk factors for poor outcome in adulthood. J Clin Psychiatry 2010;71:864-872.

35. Rogers TD, Dickson PE, Heck DH, Goldowitz D, Mittleman G, Blaha $\mathrm{CD}$. Connecting the dots of the cerebro-cerebellar role in cognitive function: neuronal pathways for cerebellar modulation of dopamine release in the prefrontal cortex. Synapse 2011;65:1204-1212.

36. Berk M, Dodd S, Kauer-Sant'anna M, Malhi GS, Bourin M, Kapczinski F, et al. Dopamine dysregulation syndrome: implications for a dopamine hypothesis of bipolar disorder. Acta Psychiatr Scand Suppl 2007:
41-49.

37. Strakowski SM, Delbello MP, Adler CM. The functional neuroanatomy of bipolar disorder: a review of neuroimaging findings. Mol Psychiatry 2005;10:105-116.

38. Strakowski SM, Adler CM, Almeida J, Altshuler LL, Blumberg HP, Chang KD, et al. The functional neuroanatomy of bipolar disorder: a consensus model. Bipolar Disord 2012;14:313-325.

39. Ring HA, Serra-Mestres J. Neuropsychiatry of the basal ganglia. J Neurol Neurosurg Psychiatry 2002;72:12-21.

40. Wessa M, Linke J. Emotional processing in bipolar disorder: behavioural and neuroimaging findings. Int Rev Psychiatry 2009;21:357-367.

41. Gabriel LR, Wu S, Kearney P, Bellve KD, Standley C, Fogarty KE, et al. Dopamine transporter endocytic trafficking in striatal dopaminergic neurons: differential dependence on dynamin and the actin cytoskeleton. J Neurosci 2013;33:17836-17846.

42. Nogues X, Micheau J, Jaffard R. Protein kinase C activity in the hippocampus following spatial learning tasks in mice. Hippocampus 1994; 4:71-77.

43. Vazquez SI, Vazquez A, Pena de Ortiz S. Different hippocampal activity profiles for PKA and PKC in spatial discrimination learning. Behav Neurosci 2000;114:1109-1118.

44. Goldapple K, Segal Z, Garson C, Lau M, Bieling P, Kennedy S, et al. Modulation of cortical-limbic pathways in major depression: treatment-specific effects of cognitive behavior therapy. Arch Gen Psychiatry 2004; 61:34-41.

45. Caetano SC, Olvera RL, Glahn D, Fonseca M, Pliszka S, Soares JC. Fronto-limbic brain abnormalities in juvenile onset bipolar disorder. Biol Psychiatry 2005;58:525-531.

46. Marchand WR, Bennett PJ, Dilda DS. Evidence for frontal-subcortical circuit abnormalities in bipolar affective disorder. Psychiatry (Edgmont) 2005;2:26-33. 